\title{
WITHDRAWAL OF LAYERED FLUID THROUGH A LINE SINK IN A POROUS MEDIUM
}

\author{
H. ZHANG and G. C. HOCKING
}

(Received 7 July 1994; revised 10 October 1994)

\begin{abstract}
The flow induced when fluid is withdrawn through a line sink from a layered fluid in a homogeneous, vertically confined porous medium is studied. A nonlinear integral equation is derived and solved numerically. For a given sink location, the shape of the interface can be determined for various values of the flow rate. The results are compared with exact solutions obtained using hodograph methods in a special case. It is found that the cusped and coning shapes of the interface can be accurately obtained for the sink situated at different depths in the fluid and the volume of flow into the sink per unit of time.
\end{abstract}

\section{Introduction}

Fluid flow in porous media is an important subject in hydrology and is of vital interest to the petroleum industry. Many fluid bodies are stratified in density. For example, the fluid in an oil reservoir may consist of a gas layer, an oil layer and a water layer, and underground water may consist of fresh water and salt water layers.

The productivity of the well and quality of the fluid withdrawn, that is, percentage of oil and fresh water, depend on the method by which they are withdrawn. For example, use of a horizontal well instead of a vertical well may lead to a change in quality of withdrawn fluid or an increase in productivity of the well. When the fluid is withdrawn from layers with different density, the withdrawn fluid will come from the layer adjacent to the point of removal until some critical value in flow rate is reached. At subcritical flow rates, the interface rises smoothly to an arch shape. At the critical flow rate, the interface is drawn upwards into a cusp shape (see Figure 5). Above the critical rate, the fluid from the other layer will break through into the sink. That will influence the quality of the oil or fresh water. Therefore, in these cases, it is of significance to understand the behaviour of the flow induced when fluid is withdrawn.

\footnotetext{
${ }^{1}$ Department of Mathematics, University of Western Australia, Nedlands, WA 6009, Australia

(C) Australian Mathematical Society, 1996, Serial-fee code 0334-2700/96
} 
The early work of determining the maximum oil flow into a well in the presence of a water cone was done by Meyer and Garder [9]. Their theoretical maximum was obtained as a function of the depth of the well below the top of the oil layer and the thickness of the oil zone at the drainage radius. Their results were used only to give an approximate, rather than an exact picture of the mechanics of a producing reservoir.

The calculation of the flow in such cases is complicated because the location of the interface is unknown. Early attempts to calculate critical flows encountered this problem. Muskat and Wyckoff [10] assumed horizontal radial flows and neglected the presence of the cone. This approximation influenced the calculated critical rates. In McCarthy's work [7] , the same problem was studied using a hodograph method to obtain analytical solutions in some special cases which were compared with the numerical solutions with a cusp obtained by a series method. Dikken [2] and Giger [3] studied this problem analytically. Giger approximated the shape of the water crest by a branch of the Dupuit parabola. This model was used to describe the water crest and its deformation at any flow up to the critical flow rate. In the study of Lucas et al. [6], a boundary-integral formulation was used to predict the steady interface shape in an unconfined porous medium and the well suction pressure was approximated by a point sink. They found that there exists a critical rate in the process.

There is a strong analogy between this work and that of studying withdrawal from water bodies with a free surface. Some other papers have considered a series of twodimensional free surface flow problems, having in common a submerged source or sink beneath a free surface, for example [1], [4], [5], [11] and [12]. Their studies have shown that steady flow having a cusped free surface which is horizontal at infinity occurs at a unique Froude number in a fluid of infinite depth, or over a range of Froude numbers for a fluid of finite depth.

In this study, an oil reservoir consisting of an oil layer confined above by an impermeable boundary and below by a water layer is investigated (see Figure 1). The interface shape of flows up to and including the critical flow, and for different locations of the well are examined. A horizontal well is represented by an infinite line sink (S), and thus end effects are neglected. This formulation of the problem has an inconsistency. Conservation of mass demands that there be a uniform horizontal flow at large distances from the sink, yet the pressure condition on the interface, given this uniform flow, would lead to an interface which approaches negative infinity as $x \rightarrow \infty$. In the earliest work of Muskat and Wyckoff [10] and Meyer and Garder [9], they limited the drainage radius. McCarthy [8] attempted to resolve this problem by assuming a constant potential boundary across the flow field at some distance from the sink in the lateral edge drive model. This method leads to a discontinuity in the shape of the interface.

In this paper, we attempt to resolve this problem by assuming that the flowing layer is confined below by an impermeable boundary, along which it is no longer necessary 
to satisfy the pressure condition. This technique may be considered to be equivalent in some ways to the method of McCarthy [8], but we believe it to be a more consistent approach. By allowing the inner edge of this lower boundary to move outward, the effect of this approximation can be gauged. This could also be considered as a problem in which a layer of oil trapped between two impermeable rock strata is exposed to a small aquifer near the well site.

Therefore, at the ends of the interface, a solid boundary (LI) is added to ensure the oil withdrawn remains vertically confined. A horizontal impermeable plane (BJ) is used to represent the solid boundary. The physical plane is shown in Figure 1.

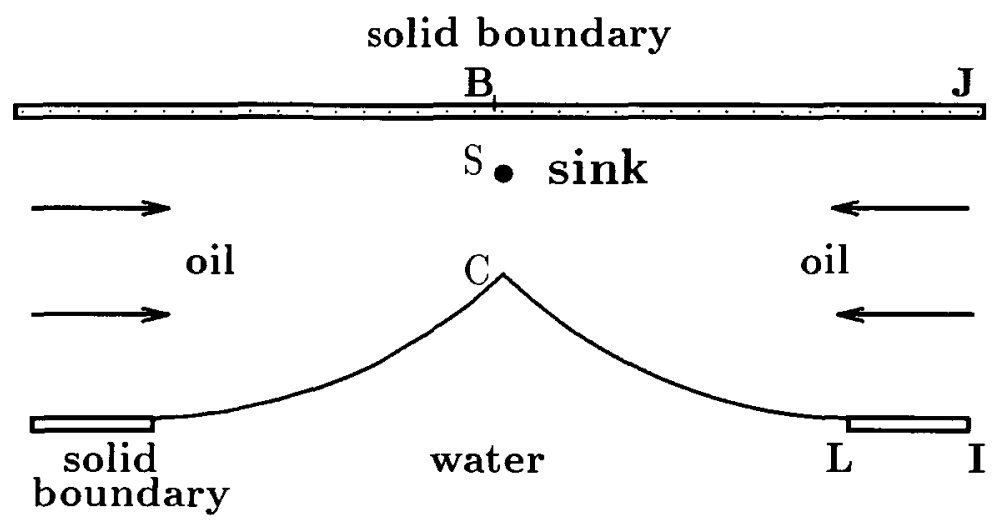

(a)

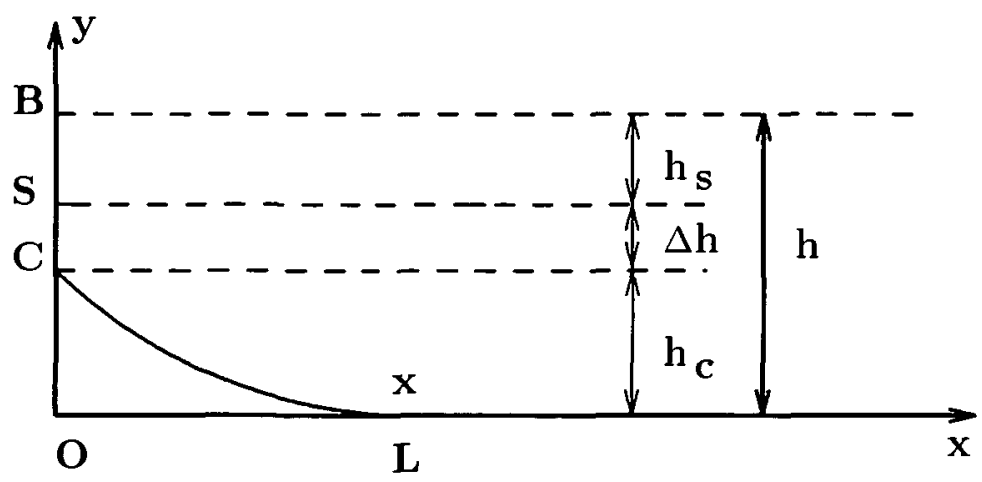

(b)

FIGURE 1. The physical plane of the confined oil reservoir. 
The hodograph method is used to obtain analytical solutions for the critical flow in the special case in which the sink is on the top impermeable plane. A nonlinear integral equation is derived and solved numerically for subcritical and critical cases with various sink locations.

\section{Problem formulation and solution}

The steady, irrotational flow of an inviscid, incompressible fluid in a homogeneous, isotropic porous medium in two dimensions is to be investigated.

Using complex variables, let the physical plane be the $z$ plane, where $z=x+i y$, and the origin is directly beneath the sink and at the level of the lower solid boundary. Darcy's equation for the two dimensional steady flow through porous media is

$$
q=-\nabla \phi
$$

where

$$
\phi=k(y+p / \gamma),
$$

and $q$ is the specific discharge vector, $\phi$ is the velocity potential function, $p$ is the pressure at a point, $y$ is the elevation above the origin, $k$ is the hydraulic conductivity and $\gamma$ is the specific gravity of the fluid. The velocity components $u$ and $v$ are related to $\phi$ by

$$
u=-\frac{\partial \phi}{\partial x}, v=-\frac{\partial \phi}{\partial y} .
$$

Assuming an incompressible fluid, and that Darcy's law is valid, leads to a problem in which Laplace's equation,

$$
\nabla^{2} \phi=0
$$

must be solved subject to conditions on the known and unknown boundaries.

Within the flow domain, there is no flow across the solid boundaries and interface, a condition which can be described by

$$
q \cdot \boldsymbol{n}=0,
$$

where $\boldsymbol{n}$ is in the direction of the normal. The lower fluid is stationary, and the entire stationary fluid region is assumed to be at a constant potential. Thus, the free surface condition along a stationary abrupt interface between a moving fluid (oil, $\gamma_{o}$ ) and a stationary fluid (water, $\gamma_{w}$ ) in an isotropic medium can be derived to be

$$
\left(\frac{\partial \phi}{\partial x}\right)^{2}+\left(\frac{\partial \phi}{\partial y}\right)^{2}+K \frac{\partial \phi}{\partial y}=0
$$


on the free surface, where $K=k\left(\gamma_{o}-\gamma_{w}\right) / \gamma_{o}$. Using $u, v$ of (2.2) and substituting into (2.4) we have

$$
u^{2}+v^{2}-K v=0
$$

on the free surface. The velocities at point $C$ and point $L$ (see Figure 1 ) are zero in the subcritical case. However, in the critical case, the velocity components at point $C$ are $u=0$ and $v=K$, and the velocity at point $\mathrm{L}$ is zero. Because of mass conversation, the flow rate must be uniform as $x \rightarrow \pm \infty$.

2.1. Analytical solution A special case in which the sink is on the upper impermeable plane of a confined oil zone (see Figure 1), is studied in an attempt to obtain an analytic solution. In the physical plane, the sink (S) on the boundary (BJ) is considered. Due to the fact that the location of the interface is unknown, it is very difficult to obtain an exact solution. Fortunately, in this case the hodograph method can be employed to attack the problem. The procedure is similar to that of Yih [13]. From (2.5), we find the free surface is a circular arc in the hodograph plane. The transformations to the hodograph plane can be obtained because all of the boundaries are straight.

Let $w=\phi(x, y)+i \psi(x, y)$ be the complex potential. Figure 2 shows the mapped planes used in this solution. Let $t=i d z / d \omega$; the free surface has been transformed into a straight line in the $t$ plane in Figure 2(b). With the transformation of Schwarz and Christoffel

$$
t=\frac{i}{\pi K} \cosh ^{-1} \zeta
$$

and

$$
w=\frac{Q}{\pi} \ln \frac{\zeta-\zeta_{A}}{\zeta-1}
$$

the $t$ plane is mapped to the $\zeta$ plane in Figure 2(c). The constant $\zeta_{A}$ is determined from $u=Q / h$ at point $I$, so that $t_{A}=(h / Q) i$, and $\zeta_{A}=\cosh (h K \pi / Q)$. Hence $z$ can be expressed in terms of $\zeta$. In fact,

$$
d z=-i t d w=\frac{Q}{\pi^{2} K}\left(\cosh ^{-1} \zeta\right)\left(\frac{1}{\zeta-\zeta_{A}}-\frac{1}{\zeta-1}\right) d \zeta .
$$

The shape of the interface is determined from (2.8) for $-\infty<\zeta \leq-1$ on the real axis. Now $\zeta$ is negative between $C$ and $L$. Hence the imaginary part of $\cosh ^{-1} \zeta$ is $\pi i$, and integration of the imaginary part of (2.8) yields

$$
y=\frac{Q}{\pi K} \ln \frac{\zeta_{A}-\zeta}{1-\zeta}
$$




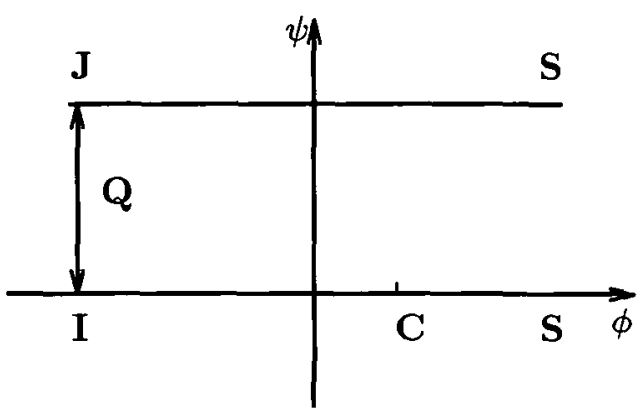

(a)

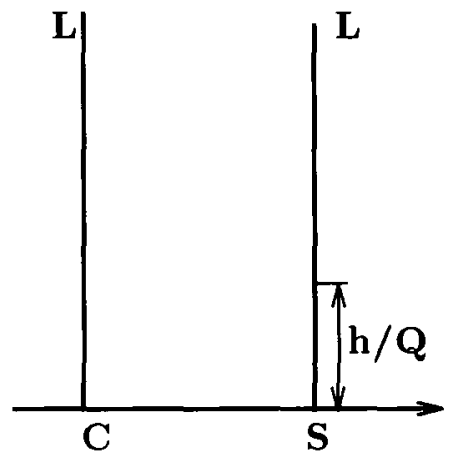

(b)

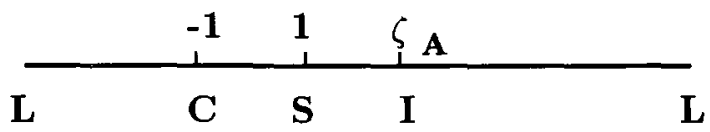

(c)

FIGURE 2. Mapped planes used in the analytic method, (a) $w$ plane (b) $t$ plane (c) $\zeta$ plane.

Letting $s=\cosh ^{-1}(\zeta)-i \pi$ for $0 \leq s \leq \infty$, and substituting $s$ into (2.8), we obtain

$$
\begin{aligned}
& x^{*}=\frac{x}{h}=\frac{1}{c \pi^{2}} \int_{s}^{\infty}\left(\frac{1}{\cosh s+\zeta_{A}}-\frac{1}{\cosh s+1}\right) s \sinh s d s \\
& y^{*}=\frac{y}{h}=\frac{1}{c \pi} \ln \frac{\cosh s+\zeta_{A}}{\cosh s+1}
\end{aligned}
$$

where $c=h K / Q$. Some examples can be seen in Figure 4. In the following analysis, the dimensionless description $y^{*}=y / h, x^{*}=x / h, h_{s}^{*}=h_{s} / h, h_{c}^{*}=h_{c} / h$ and $\Delta h^{*}=\Delta h / h$ is used. The shapes of the interface for various $c$ can be determined from (2.9) and (2.10), and we have

$$
h_{c}^{*}=\frac{1}{c \pi} \ln \frac{\zeta_{A}+1}{2} .
$$


Thus we have the important relationship

$$
c \Delta h^{*}=c-\frac{2}{\pi} \ln \left(\cosh \frac{c \pi}{2}\right) .
$$

This represents the relationship between the cusp point height and the location of the sink for various values of $c$. From (2.11), it is interesting to note that the height from the bottom boundary to the cusp point $\mathrm{C}$ is nearly equal to $h$ for very small flow rates, and for very large flow rates, $h_{c}$ approaches zero.

2.2. Numerical method As mentioned in the last section, very few analytical solutions for free surface flows in porous media can be determined because neither $\phi$ nor $y$ is known on the free surface. For instance, when the sink is moved away from the upper horizontal plane, we can no longer use the hodograph method to obtain an analytic solution. Parallel to the procedure of Hocking [4], an integral equation for this problem is to be derived and solved numerically.

The transformation

$$
e^{w \pi / Q}=\zeta
$$

maps the strip in Figure 3(a) to the lower half of the $\zeta$ plane in Figure 3(b). Let $\Omega(\zeta)=\delta(\zeta)+i \tau(\zeta)$, where $\delta(\zeta)$ is the angle between any streamline and the horizontal. The function $\Omega(\zeta)$ is defined in relation to the complex conjugate of velocity by

$$
w^{\prime}(z(\zeta))=-\frac{Q}{h} e^{-i \Omega(\zeta)},
$$

where $2 Q$ is the volume of flow into the sink per unit of time. The magnitude of velocity at any point is given by $\left|w^{\prime}(z)\right|=(Q / h) e^{\tau(\zeta)}$. The conditions that $\delta \rightarrow 0$ as $\zeta \rightarrow 1$ and $\zeta \rightarrow \zeta_{1}$ in the subcritical cases, and that $\delta \rightarrow \pi / 2$ as $\zeta \rightarrow 1$ and $\delta \rightarrow 0$ as $\zeta \rightarrow \zeta_{I}$ in the critical case, are required. The interface ( LC) corresponds to $1<\zeta<\zeta_{l}$, and the solid boundaries of the domain correspond to $-\infty<\zeta<\zeta_{b}$ and $\zeta_{l}<\zeta<\infty$. Conservation of mass requires that $\tau \rightarrow 0$ as $\zeta \rightarrow \infty$.

In order to satisfy the condition that there be no flow normal to the solid boundaries, we choose:

$$
\delta(\zeta)=\left\{\begin{array}{lll}
0 & \text { if } & -\infty<\zeta<\zeta_{b} \\
-\pi / 2 & \text { if } & \zeta_{b}<\zeta<0 \\
\pi / 2 & \text { if } & 0<\zeta<1 \\
\delta(\zeta) & \text { if } & 1<\zeta<\zeta_{l} \\
0 & \text { if } & \zeta_{1}<\zeta<\infty
\end{array}\right.
$$

The function $\Omega(\zeta)$ has weak logarithmic singularities at $\zeta=\zeta_{b}$ and $\zeta=0$, and these can be ignored. Hence, for $\operatorname{Im}(\zeta)<0$, we have,

$$
\Omega(\zeta)=-\frac{1}{2 \pi i} \oint_{-\infty}^{\infty} \frac{\Omega\left(\zeta_{0}\right)}{\zeta_{0}-\zeta} d \zeta_{0}
$$




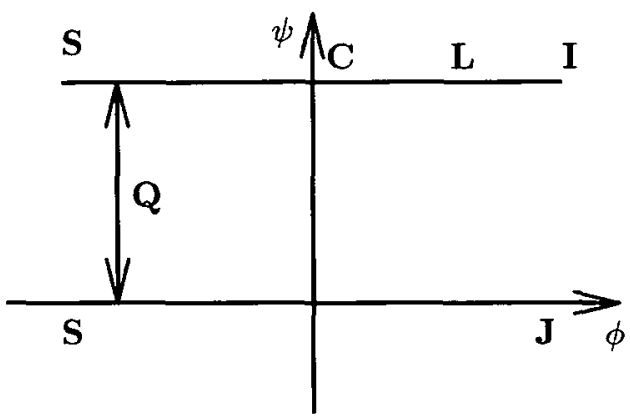

(a)

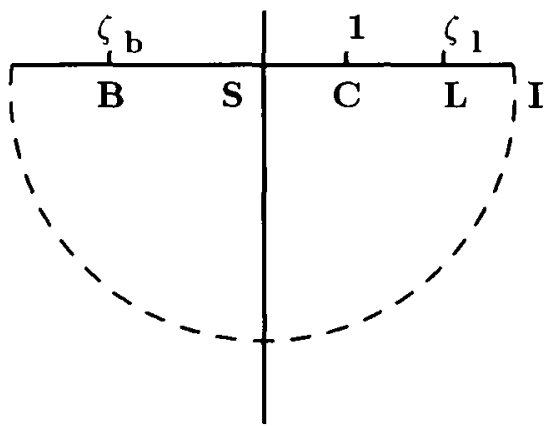

(b)

FIGURE 3. Mapped planes used in the numerical method, (a) $w$ plane (b) $\zeta$ plane.

and

$$
\begin{aligned}
\tau(\zeta) & =-\frac{1}{\pi} \int_{-\infty}^{\infty} \frac{\delta\left(\zeta_{0}\right)}{\zeta_{0}-\zeta} d \zeta_{0} \\
\delta(\zeta) & =\frac{1}{\pi} \int_{-\infty}^{\infty} \frac{\tau\left(\zeta_{0}\right)}{\zeta_{0}-\zeta} d \zeta_{0},
\end{aligned}
$$

where the integrals are of Cauchy principal value form. Substituting the known values of $\delta(\zeta)$ into (2.15) for $\tau(\zeta)$ gives

$$
\exp \tau(\zeta)=\frac{\left(\zeta-\zeta_{b}\right)^{1 / 2}(\zeta-1)^{1 / 2}}{\zeta} \exp \left(\frac{1}{\pi} \int_{1}^{\zeta_{1}} \frac{\delta\left(\zeta_{0}\right)}{\zeta_{0}-\zeta} d \zeta_{0}\right)
$$


From (2.14), we have

$$
\begin{gathered}
u=-\frac{Q}{h} e^{\tau(\zeta)} \cos \delta(\zeta), \\
v=\frac{Q}{h} e^{\tau(\zeta)} \sin \delta(\zeta),
\end{gathered}
$$

and on substituting into the free surface condition (2.5), we obtain

$$
\exp \tau(\zeta)=\frac{h K}{Q} \sin \delta(\zeta)
$$

on $1<\zeta<\zeta_{l}$. Combining (2.17) and (2.18) on the interface gives a nonlinear integral equation

$$
\frac{\left(\zeta-\zeta_{b}\right)^{1 / 2}(\zeta-1)^{1 / 2}}{\zeta} \exp \left(\frac{1}{\pi} \int_{1}^{\zeta_{1}} \frac{\delta\left(\zeta_{0}\right)}{\zeta_{0}-\zeta} d \zeta_{0}\right)=\frac{h K}{Q} \sin \delta(\zeta),
$$

on $1<\zeta<\zeta_{l}$. The values of $\delta$ on the interface are calculated from (2.19), and hence, $\tau$ can be obtained from (2.17). Using $\delta, \tau$ and integrating $d z / d \zeta$ to obtain the shape of the interface, the values of $x$ and $y$ can be written as

$$
\begin{aligned}
& x(\zeta)=-\frac{h}{\pi} \int_{\zeta_{1}}^{\zeta} \frac{e^{-\tau(\zeta)} \cos \delta(\zeta)}{\zeta} d \zeta, \\
& y(\zeta)=-\frac{h}{\pi} \int_{\zeta_{1}}^{\zeta} \frac{e^{-\tau(\zeta)} \sin \delta(\zeta)}{\zeta} d \zeta .
\end{aligned}
$$

Since the free surface condition is known, the dimensionless location of points on the interface can be written as

$$
\begin{aligned}
x^{*}(\zeta) & =\frac{1}{c \pi} \int_{\zeta}^{\zeta_{1}} \frac{1}{\zeta_{0} \tan \delta\left(\zeta_{0}\right)} d \zeta_{0} \\
y^{*}(\zeta) & =\frac{1}{c \pi} \ln \frac{\zeta_{l}}{\zeta},
\end{aligned}
$$

where $c=h K / Q$. The height of the cusped point $\mathrm{C}$ can be obtained from (2.20), being $h_{c}^{*}=(c \pi)^{-1} \ln \zeta_{1}$.

The nonlinear integral equation described by (2.19) has no closed form solution, but can be solved by computing at a set of discrete points and solving for $\delta(\zeta)$ using Newton's iteration method. Because the interface near the cusp point has the greatest change in $y$, the points are crowded near that region. Letting $\zeta=\cos ^{-2}(\theta / 2)$, for $0<\theta<\theta_{l}$, and choosing points at $N+1$ equally spaced values of $\theta$, then

$$
0<\varepsilon=\theta_{0}<\theta_{1}<\theta_{2} \cdots<\theta_{N}=\theta_{l}-\varepsilon<\theta_{l}
$$


with point spacing $\Delta \theta=\left(\theta_{N}-2 \varepsilon\right) / N$, where $\varepsilon$ is some small quantity. The integral equation (2.19) is evaluated at the mesh points $\theta_{j}$, for $j=0,1,2, \ldots, N$, with the equation rewritten with

$$
\frac{1}{\pi} \int_{1}^{\zeta_{1}} \frac{\delta\left(\zeta_{0}\right)}{\zeta_{0}-\zeta} d \zeta_{0}=\frac{1}{\pi} \int_{1}^{\zeta_{1}} \frac{\delta\left(\zeta_{0}\right)-\delta(\zeta)}{\zeta_{0}-\zeta} d \zeta_{0}+\frac{\delta(\zeta)}{\pi} \ln \frac{\zeta_{l}-\zeta}{\zeta-1}
$$

This removes the singularities in the Cauchy principal value integral and allows standard integration techniques to be used. Since $\delta(\zeta) \rightarrow 0$ as $\zeta \rightarrow 1$ and $\zeta \rightarrow \zeta_{1}$ in the subcritical case, the second term on the right hand side tends to zero as $\zeta \rightarrow 1$ and $\zeta \rightarrow \zeta_{l}$. Therefore the error associated with neglecting the end portions of the integral from $\zeta_{l}$ to $\infty$ is negligible.

In the critical case, a cusped shape is formed. Therefore, the boundary condition at $\zeta=1$ gives $\delta=\pi / 2$, but since this is the critical flow, the value of $c$ is unknown, leaving $N+1$ equations for the $N+1$ unknown $\delta_{j}, j=1,2, \ldots, N$ and $c$. Writing

$$
\delta(\zeta)=\left(\frac{\zeta_{l}^{1 / 2}-\zeta^{1 / 2}}{\zeta_{l}^{1 / 2}-1}\right) \frac{\pi}{2}+\delta_{b}(\zeta),
$$

and substituting it into the integral term of (2.19), we have

$$
\begin{array}{r}
\frac{1}{\pi} \int_{1}^{\zeta_{l}} \frac{\delta(\zeta)}{\zeta_{0}-\zeta} d \zeta_{0}=\frac{1}{\pi} \int_{1}^{\zeta_{l}} \frac{\delta_{b}(\zeta)}{\zeta_{0}-\zeta} d \zeta_{0}+\frac{\zeta_{l}^{1 / 2}}{2\left(\zeta_{l}^{1 / 2}-1\right)} \ln \frac{\zeta_{l}-\zeta}{\zeta-1} \\
+\frac{\zeta^{1 / 2}}{2\left(\zeta_{l}^{1 / 2}-1\right)}\left(\ln \frac{\zeta_{l}^{1 / 2}+\zeta^{1 / 2}}{\zeta_{l}^{1 / 2}-\zeta^{1 / 2}}-\ln \frac{1+\zeta^{1 / 2}}{1-\zeta^{1 / 2}}\right)-1
\end{array}
$$

This substitution removes the singular behaviour as $\zeta \rightarrow 1, \zeta \rightarrow \zeta_{l}$ and the procedure of solving (2.19) for $\delta_{b}$ is the same as for the subcritical case. Choosing $\zeta_{1}$ to have a particular value is effectively the same as choosing $x_{l}^{*}$, the nondimensional horizontal distance of the lower boundary away from the sink. For each value of $x_{l}^{*}$ and sink location $h_{s}^{*}$, we expect a different critical value of $c$.

This closed nonlinear system can be solved numerically using Newtonian iteration and converges to an error of less than $10^{-8}$ at the grid points usually in fewer than six iterations. In this study, the system was calculated with $N=100$ for $\theta_{l} \leq 0.99 \pi$. $N=200$ was chosen for $\theta_{l}>0.99 \pi$ to improve the accuracy of the numerical solution.

\section{Results and conclusion}

The shape of the free surface with $c=1.503$ obtained by the analytic and the numerical method are given in Figure 4, and it shows the numerical solution is in good agreement with the exact solution. 


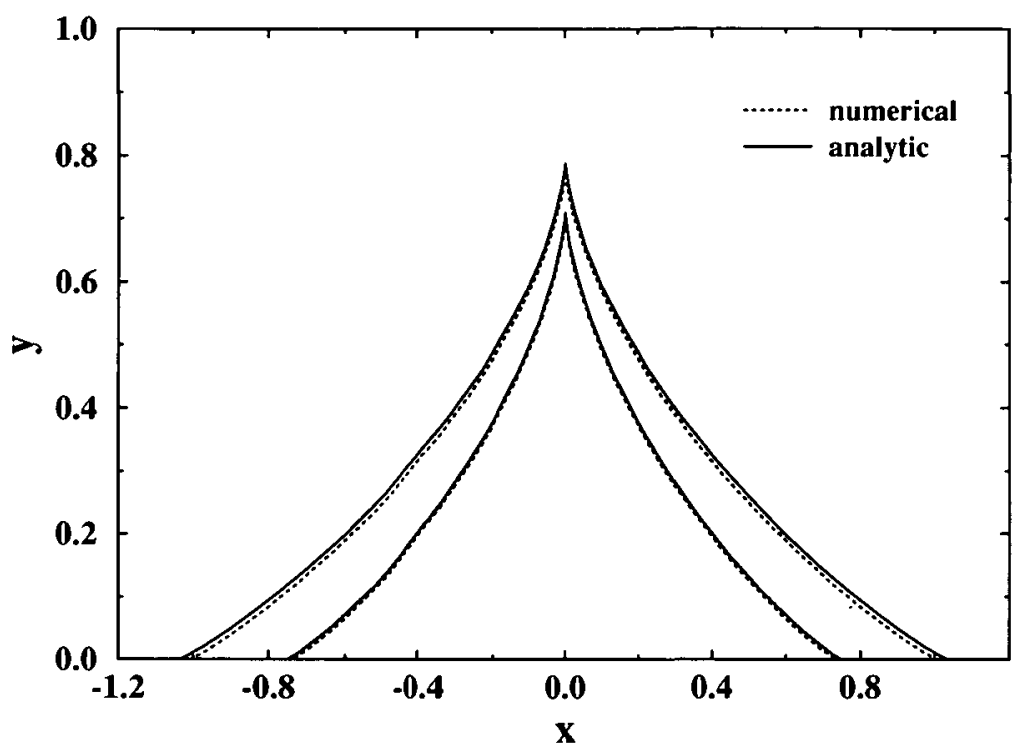

FIGURE 4. The shape of the free surface calculated using the numerical method compared with the analytic solution for the case of the sink on the upper boundary with the flow rate $c=1.504$ and $c=2.073$.

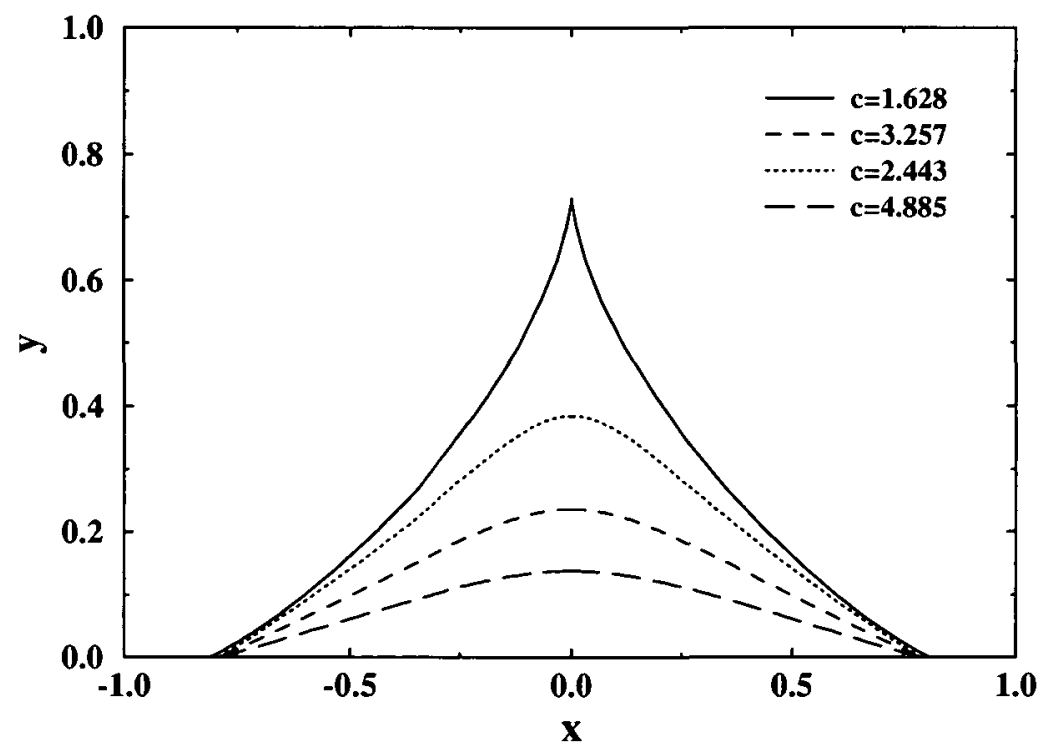

FIGURE 5. Variation of the interface shape as the flow rate is increased for the case in which the sink is on the upper boundary and the bottom boundary is fixed. 


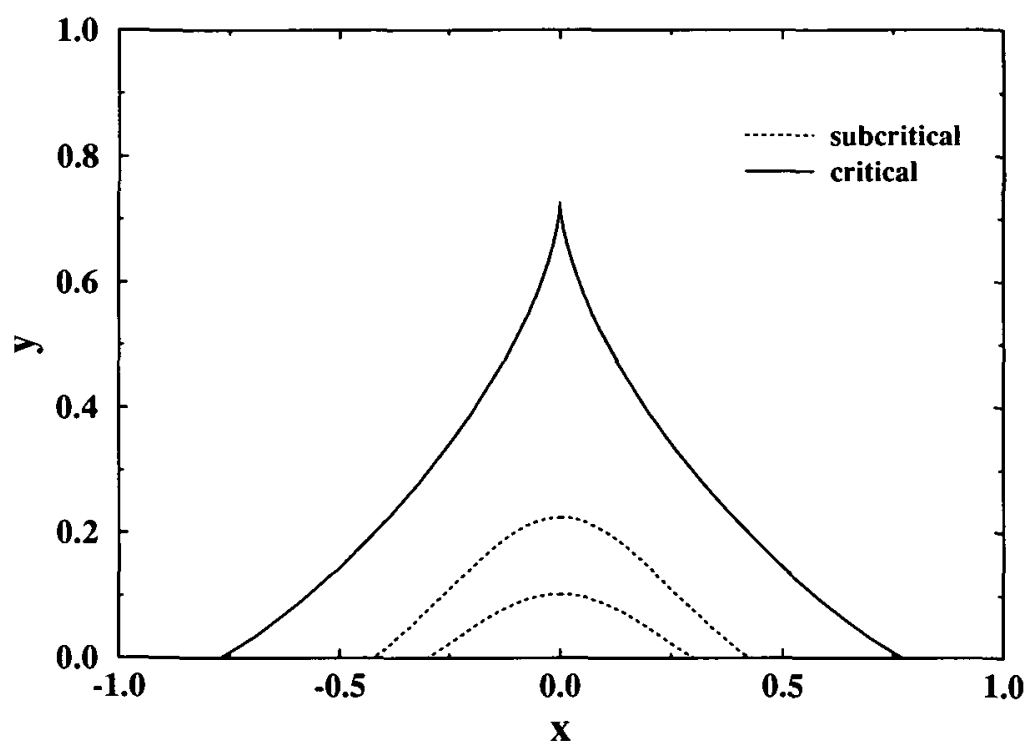

FIGURE 6. The shape of the interface calculated using the numerical method for the case of the sink on the upper boundary and a fixed flow rate $c=1.628$.

Figure 5 illustrates the effect on the shape of the interface of increasing the flow rate, where the sink location and the bottom boundary are fixed. It is found that the interface is drawn up with the increase of the flow rate until the critical rate is reached when cusping occurs. Figure 6 shows the effect on the interface shapes of moving the bottom boundary, where the sink position and flow rate are fixed, that is, how the shape changes as $x_{l}^{*}$ increases. It shows the interface shape changes from an arch shape to a cusp shape when the bottom boundary moves horizontally away from the sink.

The most important aspect of this work, however, is the behaviour of the critical flow. In the case of the sink on the impermeable plane, the comparison of $x_{l}^{*} / c$ calculated from the analytic solution with the values obtained by McCarthy [8], Giger [3] and Dikken [2] for changing values of $c$ is shown in Figure 7. In this comparison, $x_{e}^{*}$ in the papers of [8], [3] and [2], that is, the horizontal distance from the sink to the constant potential boundary, is used as $x_{l}^{*}$. Values of $c \Delta h^{*}$ obtained from the analytic solution are also shown. All values are at the critical flow rate. The analytic solution gives $x_{l}^{*} / c=0.5$ for all $c$. The curve of $c \Delta h^{*}$ increases rapidly for small $c(c<1.5)$ and then is almost horizontal at $c \Delta h^{*} \sim 0.441$ for large $c$.

The location of the sink plays an important role in the shaping of the interface. Figure 8 gives views of the cusp shapes of the interface for different locations of 


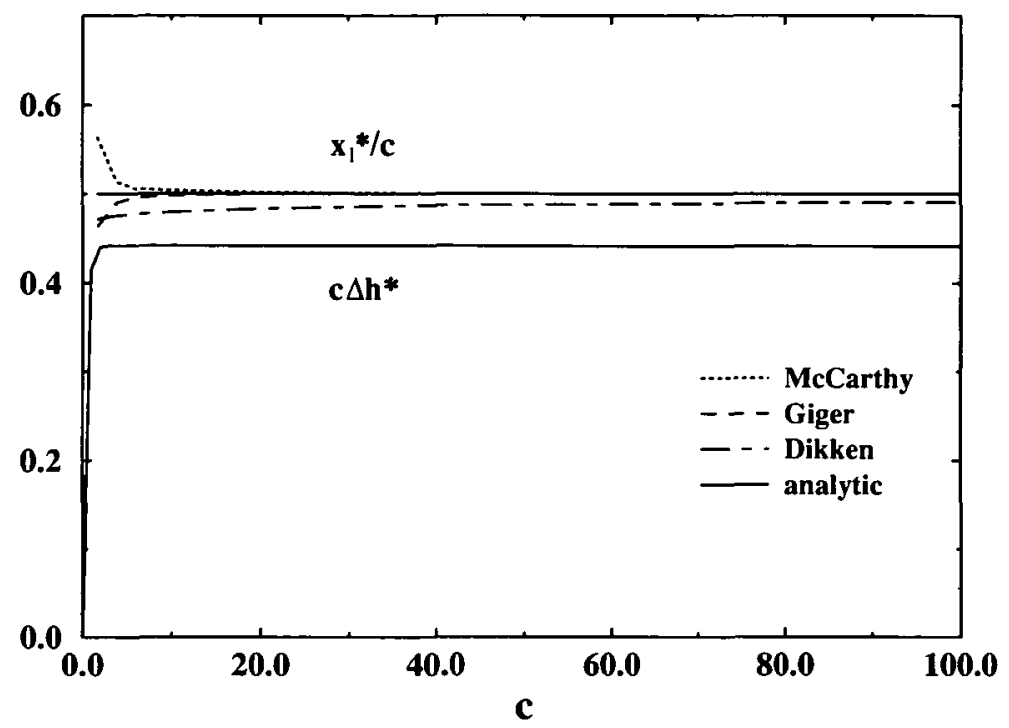

FIGURE 7. The comparison of $x_{i}^{*} / c$ calculated from the analytic solution with the values obtained by McCarthy [8], Giger [3] and Dikken [2], for changing values of $c$. Values of $c \Delta h^{*}$ obtained from the analytic solution are also shown. All values are at the critical flow rate.

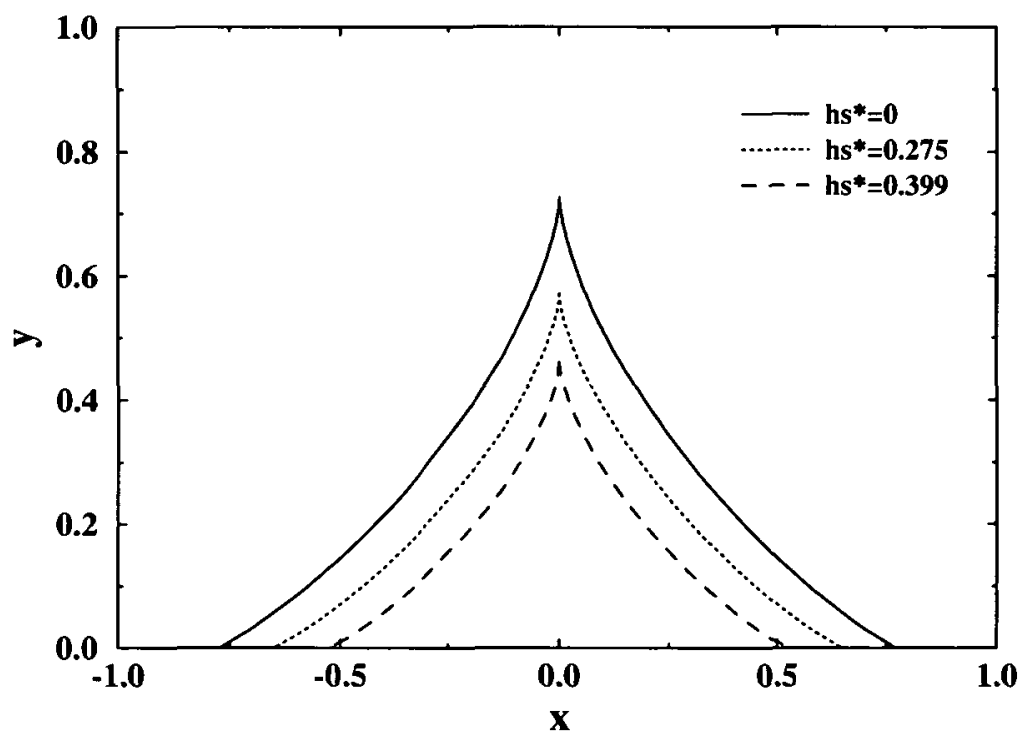

FIGURE 8. The shapes of the free surface for the case of the sink in various locations with the same flow rate $c=1.622$. 


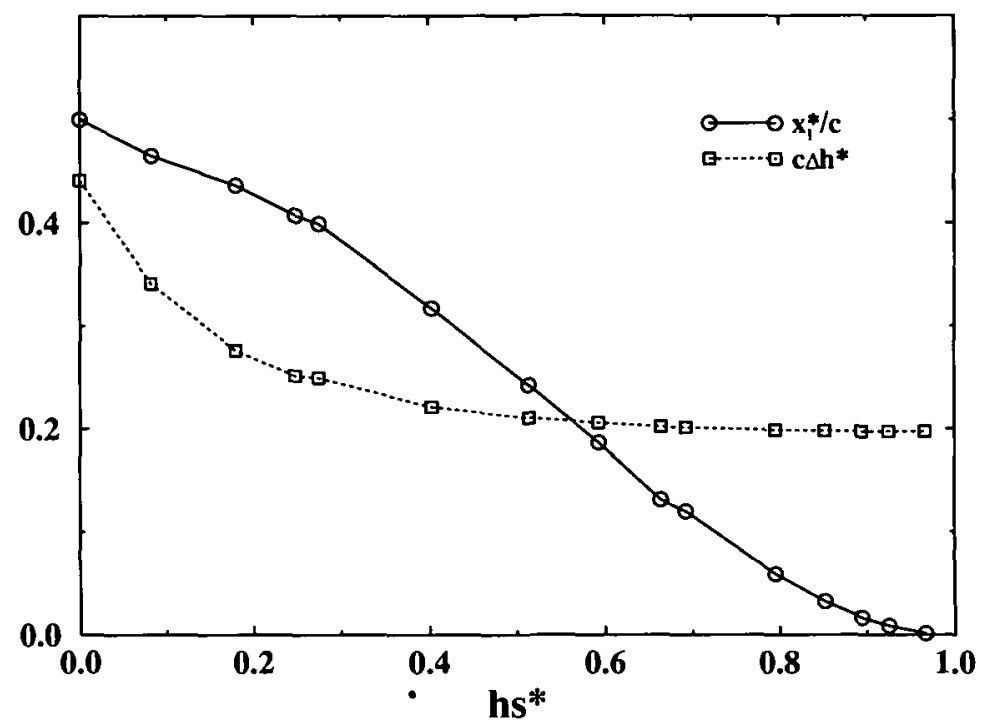

FIGURE 9. The relationship between the shape of the free surface and the location of the sink at critical flow rate.

the sink at a constant flow rate. The changes in $x_{i}^{*}$ as $h_{s}^{*}$ changes can be seen. The effects of the sink position on the interface are shown in Figure 9. It is found that $x_{l}^{*} / c$ decreases with increasing values of $h_{s}^{*}$ and $c \Delta h^{*}$ declines, but converges to a constant. This clearly shows that for a fixed value of $x_{l}^{*}$, the maximum flow rate without drawing water into the sink occurs when the sink is on the upper boundary, that is, $h_{s}^{*}=0$.

A nonlinear integral equation has been developed to compute the shape of an interface between oil and water in an oil reservoir under the influence of a withdrawal flow volume per unit of time, and for different locations of the well. This model can be used for both critical and subcritical cases. An impermeable lower boundary is used to eliminate the problem of inconsistency of the boundary conditions.

\section{References}

[1] I. L. Collings, "Two infinite-froude-number cusped free-surface due to a submerged line source or sink", J. Austral. Math. Soc. Ser. B 28 (1986) 260-270.

[2] B. J. Dikken, "Pressure drop in horizontal wells and its effect on their production performance", SPE(Society of Petroleum Engineers), Paper No. 19824 (October, 1989).

[3] F. M. Giger, "Analytic 2-D models of water cresting before breakthrough for horizontal wells", SPE Res. Eng. (November, 1989) 409-416.

[4] G. C. Hocking, "Critical withdrawal from a two-layer fluid through a line sink", J. Engng. Math. 25 (1991) 1-11. 
[5] G. C. Hocking, "Subcritical free-surface flow caused by a line source in a fluid of finite depth", $J$. Engng. Math. 26 (1992) 455-466.

[6] S. K. Lucas, J. R. Blake and A. Kucera, "A boundary-integral method applied to water coning in oil reservoirs", J. Austral. Math. Soc. Ser. B 32 (1991) 261-283.

[7] J. F. McCarthy, "Gas and water cresting towards horizontal wells", J. Austral. Math. Soc. Ser. B 35 (1993) 174-197.

[8] J. F. McCarthy, "Improved model of water cresting", J. Austral. Math. Soc. Ser. B 35 (1993) 207-222.

[9] H. I. Meyer and A. O. Garder, "Mechanics of two immisicible fluids in porous media", J. of Appl. Phys. 25 (1954) 1400-1406.

[10] M. Muskat and R. B. Wyckoff, "An approximate theory of water coning in oil production", Trans. AIME 114 (1935) 144-163.

[11] E. O. Tuck and J. M. Vanden-Broeck, "A cusp-like free-surface flow due to a submerged source or sink", J. Austral. Math. Soc. Ser. B 25 (1984) 443-450.

[12] J. M. Vanden-Broeck and J. B. Keller, "Free surface flow due to a sink", J. Fluid Mech. 175 (1987) 109-117.

[13] C. Yih, Stratified flows (Academic Press, New York, 1980). 